\title{
Predictors of Helmet CPAP Failure in COVID-19 Pneumonia: A Prospective, Multicenter, and Observational Cohort Study
}

\author{
Pierachille Santus (D), ${ }^{1,2}$ Stefano Pini $\left(D,{ }^{1,2}\right.$ Francesco Amati $\left(\mathbb{D},{ }^{3}\right.$ Marina Saad, ${ }^{1,2}$ \\ Marina Gatti, ${ }^{1,2}$ Michele Mondoni $\left(\mathbb{D},{ }^{4,5}\right.$ Francesco Tursi, ${ }^{6}$ Maurizio Rizzi, ${ }^{1}$ \\ Davide Alberto Chiumello (D), ${ }^{5,7,8}$ Valter Monzani, ${ }^{9}$ Francesco Blasi ${ }^{(D)},{ }^{10,11}$ \\ Stefano Aliberti $\mathbb{D}^{3,12}$ and Dejan Radovanovic ${ }^{1}{ }^{1}$ \\ ${ }^{1}$ Division of Respiratory Diseases, Ospedale Luigi Sacco, Polo Universitario, ASST Fatebenefratelli-Sacco, Milano, Italy \\ ${ }^{2}$ Department of Biomedical and Clinical Sciences (DIBIC), Università degli Studi di Milano, Milano, Italy \\ ${ }^{3}$ Respiratory Unit, IRCCS Humanitas Research Hospital, Rozzano, Italy \\ ${ }^{4}$ Respiratory Unit, ASST Santi Paolo e Carlo, San Paolo Hospital, Milano, Italy \\ ${ }^{5}$ Dipartimento di Scienze Della Salute, Università degli Studi di Milano, Milano, Italy \\ ${ }^{6}$ UOS Pneumologia, Ospedale Civico di Codogno, ASST Lodi, Lodi, Italy \\ ${ }^{7}$ Department of Anesthesia and Intensive Care, ASST Santi Paolo e Carlo, San Paolo University Hospital, Milano, Italy \\ ${ }^{8}$ Coordinated Research Center on Respiratory Failure, Università Degli Studi di Milano, Milano, Italy \\ ${ }^{9}$ Fondazione IRCCS Ca' Granda Ospedale Maggiore Policlinico, Department of Medicine, Acute Medical Unit, Milano, Italy \\ ${ }^{10}$ Fondazione IRCCS Ca' Granda Ospedale Maggiore Policlinico, Respiratory Unit and Cystic Fibrosis Adult Center, Milano, Italy \\ ${ }^{11}$ Università Degli Studi di Milano, Department of Pathophysiology and Transplantation, Milano, Italy \\ ${ }^{12}$ Department of Biomedical Sciences, Humanitas University, Pieve Emanuele, Milano, Italy
}

Correspondence should be addressed to Dejan Radovanovic; danko86@hotmail.com

Received 14 August 2021; Revised 6 December 2021; Accepted 24 December 2021; Published 21 January 2022

Academic Editor: Andrea S. Melani

Copyright (c) 2022 Pierachille Santus et al. This is an open access article distributed under the Creative Commons Attribution License, which permits unrestricted use, distribution, and reproduction in any medium, provided the original work is properly cited.

Background. Continuous positive airway pressure (CPAP) can be beneficial in acute respiratory failure (ARF) due to coronavirus (COVID-19) pneumonia, but delaying endotracheal intubation (ETI) in nonresponders may increase mortality. We aimed at investigating the performance of composite respiratory indexes as possible predictors of CPAP failure in ARF due to COVID-19. Methods. This was a multicenter, prospective, observational, and cohort study conducted in the respiratory units of three University hospitals in Milan and in a secondary care hospital in Codogno (Italy), on consecutive adult patients with ARF due to COVID-19 pneumonia that underwent CPAP between March 2020 and March 2021. ETI transfer to the intensive care unit or death is defined CPAP failure. Predictors of CPAP failure were assessed before T0 and 1 hour after T1 CPAP initiation and included mROX index (ratio of $\mathrm{PaO} 2 / \mathrm{FiO} 2$ to respiratory rate), alveolar-to-arterial (A-a) $\mathrm{O}_{2}$ gradient, and the HACOR (heart rate, acidosis, consciousness, oxygenation, and respiratory rate) score. Results. Three hundred and fifty four patients (mean age 64 years, $73 \%$ males) were included in the study; 136 (38.4\%) satisfied criteria for CPAP failure. A-a $\mathrm{O}_{2}$ gradient, mROX, and HACOR scores were worse in patients who failed CPAP, both at T0 and T1 ( $p<0.001$ for all parameters). The HACOR score was associated with CPAP failure (odds ratio-OR_for every unit increase in HACOR=1.361; 95\%CI: $1.103-1.680 ; p=0.004$; AUROC $=0.742 ; p<0.001)$. CPAP failure was best predicted by a threshold of 4.50 (sensitivity $=53 \%$ and specificity $=87 \%$ ). Conclusions. The HACOR score may be a reliable and early predictor of CPAP failure in patients treated for ARF in COVID19 pneumonia. 


\section{Introduction}

Continuous positive airway pressure (CPAP) is a valuable noninvasive respiratory support to treat acute hypoxic respiratory failure (hypoxic ARF) associated with coronavirus (COVID-19) pneumonia [1-3]. CPAP may avoid unnecessary endotracheal intubation (ETI) [4]; however, delaying invasive ventilation in nonresponders to CPAP may increase mortality [4]. Early and reliable predictors of CPAP failure in COVID-19 pneumonia are still lacking $[1,5,6]$.

Our aim was to investigate and compare the performance of different composite respiratory indexes in identifying CPAP failure in patients affected by COVID-19 pneumonia and ARF.

\section{Materials and Methods}

This was a multicenter, prospective, observational, and cohort study conducted in the high dependency respiratory units (HDRU) of three academic hospitals in Milan (Luigi Sacco University Hospital, Ospedale Maggiore Policlinico, and San Paolo University Hospital) and one secondary care hospital in Codogno (Italy). Consecutive adult patients with ARF due to laboratory confirmed COVID-19 pneumonia that underwent CPAP between March 2020 and March 2021 were enrolled. Exclusion criteria were ETI or death $<24$ hours from hospital admission.

CPAP was started by protocol as previously reported [1] and was delivered through high-flow generators (VitalSigns Inc; 90-140 L.min ${ }^{-1}$; MYO 3133A, Pulmodyne) using a helmet (StarMed) with an adjustable PEEP valve (VitalSigns) [1]. Anthropometrical characteristics, vital signs, and blood gas analysis were collected before CPAP (T0) and 1 hour after CPAP positioning (T1). $\mathrm{FiO}_{2}$ before CPAP was estimated by administration of oxygen via Venturi masks. Blood tests and chest X-ray were performed at T0. Hospital length of stay and all-cause in-hospital mortality were registered. According to local standard operating procedures, ceiling treatment and eligibility for ETI were judged by the treating physician and the critical care staff. Criteria for ETI and a "do-not-intubate" (DNI) order were previously reported [1]. In case of CPAP failure, noninvasive ventilation (NIV) could be instituted in the ICU based on clinical judgement and bed availability.

CPAP failure was defined as the need for ETI and/or transfer to the ICU or death in HDRU in DNI patients. Predictors of CPAP failure were assessed at T0 and T1 and included (1) mROX index (ratio of partial pressure of oxygen to inspired oxygen fraction and respiratory rate: $\mathrm{PaO}_{2} / \mathrm{FiO}_{2} / \mathrm{RR}$ ) [7], (2) alveolar-to-arterial (A-a) $\mathrm{O}_{2}$ gradient, and (3) HACOR score, a composite index ( $0-25$ points, the higher the worse) in which clinical items are scored and stratified based on severity, including heart rate (0-1), respiratory acidosis (0-4), and level of consciousness (Glasgow Coma Scale-GCS, 0-10), $\mathrm{PaO}_{2} / \mathrm{FiO}_{2}$ (0-6), and $\mathrm{RR}(0-4)$ [8].

The study (NCT04307459) followed the amended Declaration of Helsinki (2013), which was approved by the local ethical committees (No. 17263/2020, No. 345/2020, and No. 2020/ST/095), and all patients gave written informed consent.
Analyses were performed with IBM SPSS Statistics for Windows, V.23.0 (Armonk, NY). Variables were expressed as median and interquartile range (IQR) or means and standard deviation (SD), according to their distribution assessed with the Shapiro-Wilk test. Independent groups $T$ test, Chi-square, or Mann-Whitney tests were used to compare CPAP successes and failures. A logistic regression analysis was performed to investigate determinants of CPAP failure. The area under the receiver operating characteristic curve (AUROC) was calculated for CPAP failure predictors at T0 and T1. Tests were two sided and statistical significance was taken at $p<0.05$.

\section{Results}

Three hundred and fifty four patients (mean age 64 years, $73 \%$ males) were included in the study, of which $136(38.4 \%)$ satisfied criteria for CPAP failure (Table 1). Fifty-one (14.4\%) patients received a DNI order, of which $32(62.7 \%)$ died. Ninety seven $(27.4 \%)$ patients were transferred to the ICU, of which $64(65.8 \%)$ were intubated. Seven (7.2\%) patients failed CPAP and received invasive mechanical ventilation while in the HDRU (Table 1). In-hospital mortality was $27.1 \%$ while ICU mortality was $66 \%$. Among patients who were not directly intubated in the ICU, $20.6 \%$ received NIV and all eventually undergone ETI. Mortality in patients exposed to NIV did not significantly differ compared with patients intubated after CPAP failure $(60.0 \%$ vs. $58.4 \%$, $p=0.742$ ).

Patients that failed CPAP were older, more likely to have chronic comorbidities, and had worse clinical severity, hypoxemia, $\mathrm{A}-\mathrm{a} \mathrm{O}_{2}$ gradient, mROX, and HACOR scores at T0 than patients who succeeded it, respectively, 326 (96-604) $\mathrm{mmHg}$ vs. 179 (56-315) $\mathrm{mmHg}, 4.3$ (2.3-6.5) $\mathrm{mmHg} / \mathrm{bpm}$ vs. 7.3 (4.8-11.1) $\mathrm{mmHg} / \mathrm{bpm}$, and 6 (3.0-6.3) vs. 2 (0-5.0) (Table 1). After CPAP initiation, respiratory parameters tended to improve in both groups, but were still deteriorated in patients that failed CPAP (Table 1).

The logistic regression analysis, including age, history of ischemic heart disease and chronic pulmonary disease, D-dimer and C-reactive protein at admission, $\mathrm{PaO}_{2} / \mathrm{FiO}_{2}$, $\mathrm{RR}, \mathrm{mROX}, \mathrm{A}-\mathrm{a} \mathrm{O}_{2}$ gradient, and HACOR score at $\mathrm{T} 1$ as independent variables, showed that the only parameter significantly associated with CPAP failure was the HACOR score (odds ratio-OR-for every unit increase in HACOR $=1.361 ; \quad 95 \% \mathrm{CI}: 1.103-1.680 ; \quad p=0.004$; AUROC $=0.742 ; p<0.001$ ) (Figure 1). The threshold that best discriminated CPAP failure was 4.50 (sensitivity, i.e., true positive rate $=53 \%$; specificity, i.e., true negative rate$=87 \%)$. The results did not change excluding DNI patients or after performing a sensitivity analysis that sequentially removed data relative to each participating center (data not shown).

\section{Discussion}

To date, only a limited number of studies explored predictors for CPAP failure in patients with COVID-19 pneumonia, with inconsistent results. CPAP failure was shown to be 
TABLE 1: Clinical characteristics at admission and after CPAP positioning and predictors of CPAP failure

\begin{tabular}{|c|c|c|c|c|}
\hline Characteristics $(n=354)$ & $\begin{array}{l}\text { All CPAP patients } \\
\quad(n=354)\end{array}$ & $\begin{array}{l}\text { CPAP success } \\
\quad(n=218)\end{array}$ & $\begin{array}{l}\text { CPAP failure } \\
\quad(n=136)\end{array}$ & $p$ value $^{\mathrm{a}}$ \\
\hline Age (years) & $64(56-73)$ & $62(54-71)$ & $69(60-76)$ & $<0.001$ \\
\hline Males, $n(\%)$ & $260(73.4)$ & $164(75.2)$ & $96(70.6)$ & 0.336 \\
\hline Hypertension, $n(\%)$ & $164(46.3)$ & $98(45)$ & $66(48.5)$ & 0.512 \\
\hline Diabetes mellitus, $n(\%)$ & $80(22.6)$ & $45(20.6)$ & $35(25.7)$ & 0.275 \\
\hline Ischemic heart disease, $n(\%)$ & $56(15.8)$ & $21(9.6)$ & $35(25.7)$ & $<0.001$ \\
\hline $\mathrm{COPD}, n(\%)$ & $25(7.1)$ & $10(4.6)$ & $15(11)$ & 0.021 \\
\hline DNI, $n(\%)$ & $51(14.4)$ & $19(8.7)$ & $32(23.5)$ & $<0.001$ \\
\hline \multicolumn{5}{|l|}{ In-hospital treatment $(n=272)$} \\
\hline Systemic corticosteroids, $n(\%)$ & $190(69.9)$ & $107(69.0)$ & $83(70.9)$ & 0.734 \\
\hline Tocilizumab, $n(\%)$ & $45(12.7)$ & $25(11.5)$ & $20(9.2)$ & 0.682 \\
\hline LMWH, prophylactic, $n(\%)$ & $103(37.9)$ & $67(43.2)$ & $36(30.8)$ & 0.067 \\
\hline LMWH, therapeutic, $n(\%)$ & $156(57.4)$ & $84(54.2)$ & $72(61.5)$ & 0.084 \\
\hline \multicolumn{5}{|l|}{ Outcomes $(n=354)$} \\
\hline Time to CPAP start, days & $2(1-4)$ & $2(1-4)$ & $1(0-3)$ & 0.086 \\
\hline CPAP duration, days & $6(3-9)$ & $6(4-10)$ & $4(3-8)$ & $<0.001$ \\
\hline Hospital length of stay, days & $16(11-25)$ & $18(13-26)$ & $11(6-20)$ & $<0.001$ \\
\hline Admitted to ICU, $n(\%)$ & $97(27.4)$ & 0 & $97(71.3)$ & N.A. \\
\hline Received NIV after CPAP failure in ICU, $n(\%)$ & $20(20.6)$ & 0 & $20(20.6)$ & N.A. \\
\hline ETI, $n(\%)$ & $71(20.1)$ & 0 & $71(52.2)$ & N.A. \\
\hline ETI after NIV, $n(\%)$ & $20(100)$ & 0 & $20(100)$ & N.A. \\
\hline ETI not moved to ICU, $n(\%)$ & $7(2.0)$ & 0 & $7(5.1)$ & N.A. \\
\hline In-hospital mortality, $n(\%)$ & $96(27.1)$ & 0 & $96(70.6)$ & N.A. \\
\hline ICU mortality, $n(\%)$ & $64(66.0)^{9}$ & 0 & $65(66.0)^{9}$ & N.A. \\
\hline Mortality after positioning NIV, $n(\%)$ & $12(60.0)$ & 0 & $12(60.0)$ & N.A. \\
\hline Mortality among DNI, $n(\%)$ & $32(9.0)$ & 0 & $32(23.5)$ & N.A. \\
\hline \multicolumn{5}{|l|}{ Clinical variables at admission } \\
\hline White blood cells $\left(\times 10^{6} / \mathrm{L}\right)$ & $7.37(5.50-10.11)$ & $7.06(5.4-9.6)$ & $8.5(5.7-11.6)$ & 0.019 \\
\hline Platelets $\left(\times 10^{6} / \mathrm{L}\right)$ & $214(165-298)$ & $221(172-303)$ & $201(148-284)$ & 0.052 \\
\hline D-dimer $(\mu \mathrm{g} / \mathrm{L}$ FEU $)$ & $854(489-1425)$ & 709 (425-1199) & $1084(642-2456)$ & $<0.001$ \\
\hline $\mathrm{CRP}(\mathrm{mg} / \mathrm{L})$ & $34(12-114)$ & $31(11-92)$ & $39(15-143)$ & 0.039 \\
\hline Body temperature $\left({ }^{\circ} \mathrm{C}\right)$ & $37.1(36.4-38)$ & $37(36.4-37.8)$ & $37.4(36.5-38)$ & 0.067 \\
\hline \multicolumn{5}{|l|}{ CPAP parameters } \\
\hline $\operatorname{PEEP}\left(\mathrm{cmH}_{2} \mathrm{O}\right)$ & $10(7.5-10)$ & $10(7.5-10)$ & $10(8-12)$ & 0.002 \\
\hline $\mathrm{FiO}_{2}(\%)$ & $60(50-60)$ & $60(50-60)$ & $60(60-70)$ & $<0.001$ \\
\hline \multicolumn{5}{|c|}{ Respiratory and clinical parameters before CPAP positioning (T0) } \\
\hline $\mathrm{pH}$ & $7.47(7.45-7.50)$ & $7.47(7.45-7.50)$ & $7.48(7.45-7.51)$ & 0.808 \\
\hline $\mathrm{PaCO}_{2}(\mathrm{mmHg})$ & $33(29-37)$ & $33(30-37)$ & $32(29-35)$ & 0.058 \\
\hline $\mathrm{PaO}_{2} / \mathrm{FiO}_{2}(\mathrm{mmHg})$ & $161(100-252)$ & $194(122-273)$ & $110(68-181)$ & $<0.001$ \\
\hline $\mathrm{A}-\mathrm{a} \mathrm{O}_{2}$ gradient $(\mathrm{mmHg})$ & $239(61-432)$ & $179(56-315)$ & $326(96-604)$ & $<0.001$ \\
\hline Respiratory rate (bpm) & $27(23-30)$ & $25(22-30)$ & $28(24-34)$ & $<0.001$ \\
\hline Glasgow coma scale* & $15(15-15)$ & $15(15-15)$ & $15(15-15)$ & 0.001 \\
\hline ROX index (\%/bpm) & $8(4.4-14.8)$ & $9.5(5.9-15.9)$ & $5.4(3.3-11.1)$ & $<0.001$ \\
\hline mROX index $(\mathrm{mmHg} / \mathrm{bpm})$ & $6.1(3.6-9.7)$ & $7.3(4.8-11.1)$ & $4.3(2.3-6.5)$ & $<0.001$ \\
\hline HACOR score & $4.0(0-6.0)$ & $2(0-5.0)$ & $6(3.0-6.3)$ & $<0.001$ \\
\hline \multicolumn{5}{|c|}{ Respiratory and clinical parameters 1 hour after CPAP positioning (T1) } \\
\hline $\mathrm{pH}$ & $7.46(7.44-7.49)$ & $7.46(7.44-7.49)$ & $7.47(7.43-7.49)$ & 0.812 \\
\hline $\mathrm{PaCO}_{2}(\mathrm{mmHg})$ & $36(33-40)$ & $37(34-40)$ & $35(31-39)$ & 0.004 \\
\hline $\mathrm{PaO}_{2} / \mathrm{FiO}_{2}(\mathrm{mmHg})$ & $188(126-251)$ & $207(161-265)$ & $132(108-220)$ & $<0.001$ \\
\hline $\mathrm{A}-\mathrm{a} \mathrm{O}_{2}$ gradient $(\mathrm{mmHg})$ & $254(196-308)$ & $230(183-279)$ & $302.6(234-330)$ & $<0.001$ \\
\hline Respiratory rate (bpm) & $24(20-28)$ & $24(20-28)$ & $26(22-30)$ & $<0.001$ \\
\hline Glasgow coma scale** & $15(15-15)$ & $15(15-15)$ & $15(15-15)$ & 0.011 \\
\hline ROX index (\%/bpm) & $6.9(5.6-8.5)$ & $7.6(6.2-9.2)$ & $6.2(4.7-7.7)$ & $<0.001$ \\
\hline mROX index $(\mathrm{mmHg} / \mathrm{bpm})$ & $7.6(5.1-10.9)$ & $8.8(6.7-12.1)$ & $5.8(3.8-8.9)$ & $<0.001$ \\
\hline HACOR score & $2(0-5)$ & $1(0-3)$ & $5(2-6)$ & $<0.001$ \\
\hline
\end{tabular}

Data are presented as medians (interquartile range), unless otherwise stated. DNI, "do-not-intubate" order; $\mathrm{A}-\mathrm{a} \mathrm{O}_{2}$ gradient, alveolar-arterial oxygen gradient; bpm, breaths per minute; $\mathrm{CI}$, confidence interval; CPAP, continuous positive airway pressure; CRP, C-reactive protein (upper limit of normal = $10 \mathrm{mg} / \mathrm{L}$ ); FEU, fibrinogen equivalent units; GCS, Glasgow coma scale; $\mathrm{PaO}_{2}$, arterial partial pressure of oxygen; $\mathrm{PaCO}_{2}$, arterial partial pressure of carbon dioxide; ROX index, $\mathrm{SpO}_{2} / \mathrm{FiO}_{2} /$ respiratory rate; mROX index, modified $\mathrm{ROX}$ index $\left(\mathrm{PaO}_{2} / \mathrm{FiO}_{2} /\right.$ respiratory rate); HACOR, heart rate, acidosis ( $\left.\mathrm{pH}\right)$, consciousness (Glasgow coma scale: GCS), oxygenation, and respiratory rate; AUROC, area under the receiver operating characteristic curve. ${ }^{\mathrm{a} C o m p a r i s o n}$ between CPAP success and CPAP failure. * denotes seven (3.2\%) patients in the success group vs. $16(11.8 \%)$ patients in the failure group had a GSC $<15 .{ }^{* *}$ denotes eight (3.7\%) patients in the success group vs. 15 (11\%) patients in the failure group had a GSC $<15$. 'denotes among patients admitted to ICU. 


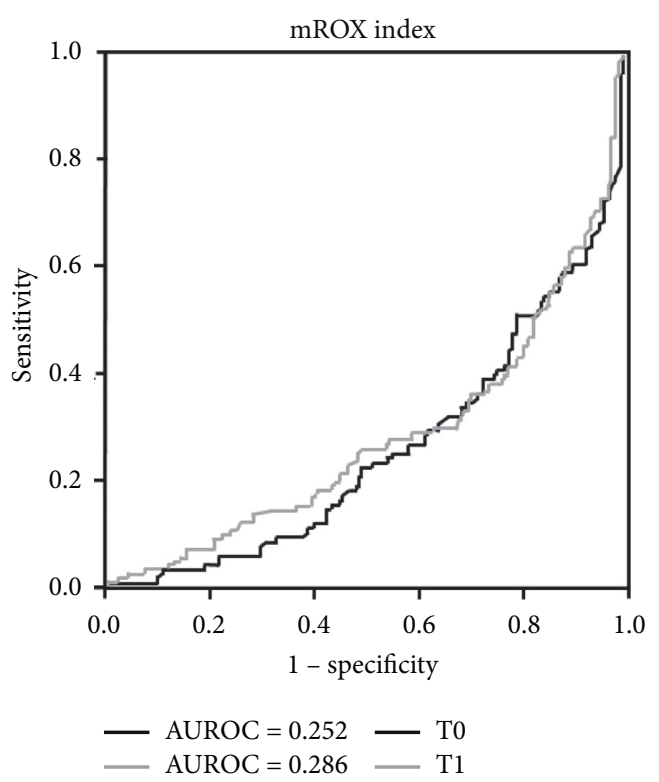

(a)

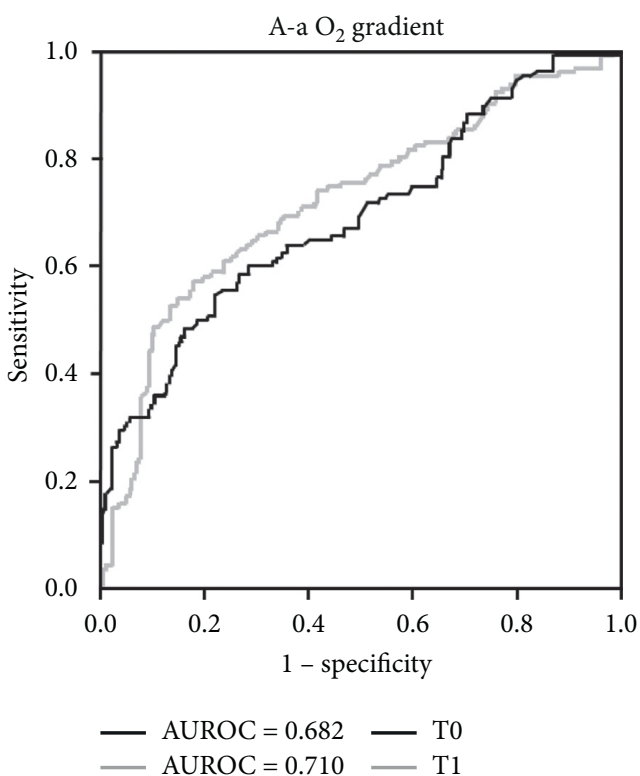

(b)

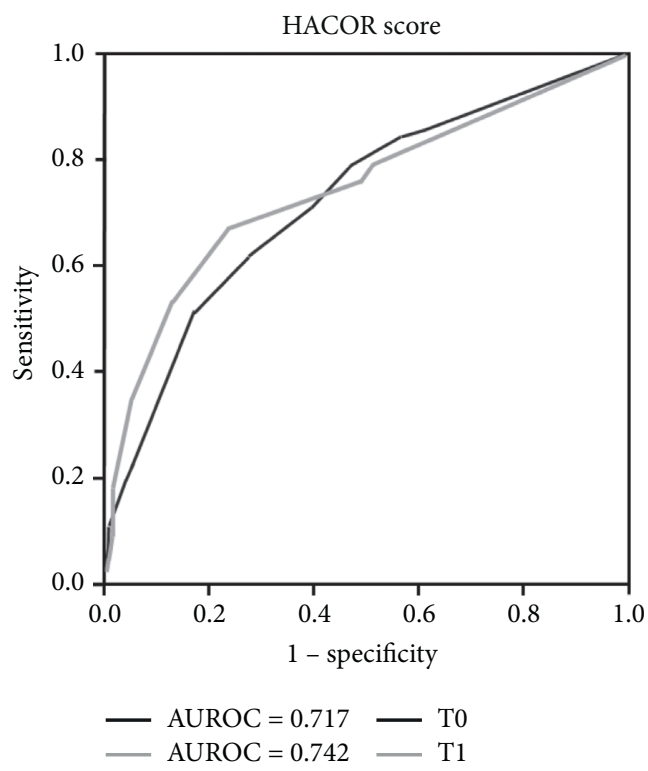

(c)

FIgURE 1: Accuracy of predictors of CPAP failure. Receiver operating characteristic (ROC) curves with areas under the ROC curves showing the performance for mROX index (a), A-a $\mathrm{O}_{2}$ gradient (b), and HACOR score (c) in predicting CPAP failure at T0 (black lines) and T1 (grey lines). $\operatorname{mROX}$ index $=$ modified $\mathrm{ROX}$ index $\left(\mathrm{PaO}_{2} / \mathrm{FiO}_{2} /\right.$ respiratory rate); $\mathrm{A}-\mathrm{a} \mathrm{O}_{2}$ gradient=alveolar-arterial oxygen gradient; $\mathrm{HACOR}=$ heart rate, acidosis $(\mathrm{pH})$, consciousness (Glasgow coma scale: GCS), oxygenation, and respiratory rate score; AUROC = area under the receiver operating characteristic curve.

independently predicted by pneumonia severity and interleukin-6 level at admission [1], by the presence of hypertension [5], or, more recently, by a combination of age, gender, lactic dehydrogenase, and change in $\mathrm{PaO}_{2} / \mathrm{FiO}_{2}$ after CPAP positioning (relative risk 95\%CI: 0.998, 0.996-0.999) [6].

The mROX index, an evolution of the ROX index (ratio of the percentage of arterial oxygen saturation, measured via a pulse oximeter, and respiratory rate: $\mathrm{SaO}_{2} / \mathrm{FiO}_{2} / \mathrm{RR}$ ) [9], is a predictor of failure of high-flow nasal cannula (HFNC) in patients with ARF [7]. The ROX index, but not the mROX index, has been recently investigated as a potential predictor of HFNC failure in patients with COVID-19 pneumonia [10].

The HACOR score is a validated predictor of failure of noninvasive ventilation (NIV) and HFNC, both in acute respiratory failure $[8,11]$ and in respiratory failure in patients with COPD [12].

In patients with ARF, CPAP improves both hypoxemia and work of breathing [2]. In COVID-19 pneumonia, however, the application of CPAP has shown to improve oxygenation to a variable extent, with unclear effects on 
patients' outcomes $[1,5,13,14]$, suggesting that the $a$ priori estimation of CPAP efficacy judged solely on the severity of hypoxia may lead to an underestimation of CPAP potential positive effects [15-17].

The HACOR score could help to evaluate response to CPAP because it is a composite index that includes surrogates of gas exchange efficiency $\left(\mathrm{PaO}_{2} / \mathrm{FiO}_{2}\right)$, clinical severity (heart rate, GCS), and respiratory distress ( $\mathrm{RR}, \mathrm{pH})$, thus overcoming the limits of using just a potentially unreliable index like $\mathrm{PaO}_{2} / \mathrm{FiO}_{2}$ [15]. The mROX also combines a noninvasive surrogate of respiratory distress (RR) and gas exchange efficiency $\left(\mathrm{PaO}_{2} / \mathrm{FiO}_{2}\right)$. The $\mathrm{mROX}$, as a difference from the ROX index, uses $\mathrm{PaO}_{2}$ instead of $\mathrm{SaO}_{2}$ to estimate the oxygenation in patients with ARF; this parameter requires an arterial blood gas analysis (ABG) to be performed, thus is more invasive than just relying on pulse oximeter, but avoids the intrinsic inaccuracies related to measuring and interpreting $\mathrm{SaO}_{2}$ [15].

We also observed that patients that failed CPAP had persistently elevated $\mathrm{A}-\mathrm{a} \mathrm{O}_{2}$ gradient and respiratory rate despite the ventilatory support, sustaining the hypothesis that severe ventilation/perfusion mismatch and consequent profound hypoxemia may cause exhaustion and lead to ETI. In this view, Corradi et al. have recently conducted a study that suggested that the diaphragmatic performance, as assessed by ultrasonography, which may be an accurate predictor of CPAP failure in patients with ARF due to COVID-19 [18].

Our results suggest that HACOR score predicts early CPAP failure in COVID-19 patients with high specificity, which could be of high clinical interest for resource allocation and monitoring optimization. Interestingly, patients that failed CPAP had a median HACOR score of 5, which corresponds to the threshold that predicted NIV failure in the original validation study [8]. Instead, the limited sensitivity could be ascribed to the low number of patients having positive scores in the " $\mathrm{pH}$ " and "GCS" items in our population.

The severity of respiratory failure and the rate of CPAP failure were comparable with previous studies $[1,5,6]$, and CPAP failure was not influenced by timing of initiation or by in-hospital treatment. Helmet NIV has been shown to be superior to HFNC in avoiding ETI in patients with severe COVID-19 pneumonia [19]. Moreover, recent evidence suggested that a noninvasive support escalation strategy based on a NIV trial after CPAP failure might reduce mortality and avoid unnecessary ETI, while not deteriorating patients' clinical status or delaying intubation [20].

The present study has limitations. The scores should be tested at different time points, to assess the variability of their performance after CPAP positioning. Changes in patients' severity and healthcare system preparedness during the observation year may have influenced the clinical outcomes [21]. Finally, the combined primary end point of the study may have biased the accuracy of predictive indexes (e.g., mortality in DNI patients). The sensitivity analyses, however, sustained the robustness of the results. Further studies are needed to prospectively validate the score in COVID-19 patients.

\section{Conclusions}

In conclusion, the HACOR score may be a reliable and early bedside predictor of CPAP failure in patients with ARF due to COVID-19 and could help identifying patients at higher risk of unfavorable outcome and that might benefit from an early targeted pharmacological intervention, a prompt escalation, to noninvasive ventilation or from the institution of invasive mechanical ventilation.

\section{Data Availability}

The data used to support the findings of the study are available from the corresponding author upon request.

\section{Conflicts of Interest}

The authors declare that there are no conflicts of interest regarding the publication of this article.

\section{Acknowledgments}

The authors wish to thank all the patients and the healthcare personnel involved in the study and during the COVID-19 pandemic.

\section{References}

[1] S. Aliberti, D. Radovanovic, F. Billi et al., "Helmet CPAP treatment in patients with COVID-19 pneumonia: a multicentre cohort study," European Respiratory Journal, vol. 56, no. 4, Article ID 2001935, 2020.

[2] D. Radovanovic, M. Rizzi, S. Pini, M. Saad, D. A. Chiumello, and P. Santus, "Helmet CPAP to treat acute hypoxemic respiratory failure in patients with COVID-19: a management strategy proposal," Journal of Clinical Medicine, vol. 9, no. 4, p. 1191, 2020.

[3] D. Radovanovic, S. Coppola, E. Franceschi et al., "Mortality and clinical outcomes in patients with COVID-19 pneumonia treated with non-invasive respiratory support: a rapid review," Journal of Critical Care, vol. 65, pp. 1-8, 2021.

[4] R. Vaschetto, F. Barone-Adesi, F. Racca et al., "Outcomes of COVID-19 patients treated with continuous positive airway pressure outside the intensive care unit," ERJ Open Research, vol. 7, no. 1, pp. 00541-02020, 2021.

[5] C. Brusasco, F. Corradi, A. Di Domenico et al., "Continuous positive airway pressure in COVID-19 patients with moderate-to-severe respiratory failure," European Respiratory Journal, vol. 57, no. 2, Article ID 2002524, 2021.

[6] N. De Vita, L. Scotti, G. Cammarota et al., "Predictors of intubation in COVID-19 patients treated with out-of-ICU continuous positive airway pressure," Pulmonology, vol. 2021, no. 21, pp. 2-7, 2021.

[7] H. M. R. Karim and A. M. Esquinas, "Success or failure of high-flow nasal oxygen therapy: the ROX index is good, but a modified ROX index may Be better," American Journal of Respiratory and Critical Care Medicine, vol. 200, no. 1, pp. 116-117, 2019.

[8] J. Duan, X. Han, L. Bai, L. Zhou, and S. Huang, "Assessment of heart rate, acidosis, consciousness, oxygenation, and respiratory rate to predict noninvasive ventilation failure in hypoxemic patients," Intensive Care Medicine, vol. 43, no. 2, pp. 192-199, 2017. 
[9] O. Roca, J. Messika, B. Caralt et al., "Predicting success of high-flow nasal cannula in pneumonia patients with hypoxemic respiratory failure: the utility of the ROX index," Journal of Critical Care, vol. 35, pp. 200-205, 2016.

[10] M. L. Vega, R. Dongilli, G. Olaizola et al., "COVID-19 Pneumonia and ROX index: time to set a new threshold for patients admitted outside the ICU," Pulmonology, vol. S2531S0437, no. 21, 2021.

[11] D. M. Magdy and A. Metwally, "The utility of HACOR score in predicting failure of high-flow nasal oxygen in acute hypoxemic respiratory failure," Advances in Respiratory Medicine, vol. 89, no. 1, pp. 23-29, 2021.

[12] J. Duan, S. Wang, P. Liu et al., "Early prediction of noninvasive ventilation failure in COPD patients: derivation, internal validation, and external validation of a simple risk score," Annals of Intensive Care, vol. 9, no. 1, p. 108, 2019.

[13] N. De Vita, L. Scotti, G. Cammarota, and for Covid-19 Eastern Piedmont Network, "Predictors of intubation in COVID-19 patients treated with out-of-ICU continuous positive airway pressure," Pulmonology, vol. S2531-0437, no. 21, pp. 2-7, 2021.

[14] F. Amati, S. Aliberti, S. Misuraca et al., "Lung recruitability of COVID-19 pneumonia in patients undergoing helmet CPAP," Archivos de Bronconeumología, vol. 57, no. Suppl 1, pp. 92-94, 2021.

[15] M. J. Tobin, A. Jubran, and F. Laghi, "PaO2/FIO2ratio: the mismeasure of oxygenation in COVID-19," European Respiratory Journal, vol. 57, no. 3, Article ID 2100274, 2021.

[16] M. J. Tobin, A. Jubran, and F. Laghi, "Noninvasive strategies in COVID-19: epistemology, randomised trials, guidelines, physiology," European Respiratory Journal, vol. 57, no. 2, Article ID 2004247, 2021.

[17] P. Santus, D. Radovanovic, L. Saderi et al., "Severity of respiratory failure at admission and in-hospital mortality in patients with COVID-19: a prospective observational multicentre study," BMJ Open, vol. 10, no. 10, Article ID e043651, 2020.

[18] F. Corradi, L. Vetrugno, D. Orso et al., "Diaphragmatic thickening fraction as a potential predictor of response to continuous positive airway pressure ventilation in covid-19 pneumonia: a single-center pilot study," Respiratory Physiology \& Neurobiology, vol. 284, Article ID 103585, 2021.

[19] D. L. Grieco, L. S. Menga, M. Cesarano et al., "Effect of helmet noninvasive ventilation vs high-flow nasal oxygen on days free of respiratory support in patients with COVID-19 and moderate to severe hypoxemic respiratory failure: the HENIVOT randomized clinical trial," JAMA, vol. 325, no. 17, pp. 1731-1743, 2021.

[20] S. Coppola, P. Santus, G. Sotgiu et al., "Feasibility and clinical outcomes of a step up noninvasive respiratory support strategy in patients with severe COVID-19 pneumonia," Journal of Clinical Medicine, vol. 10, no. 22, p. 5444, 2021.

[21] D. Radovanovic, S. Pini, E. Franceschi et al., "Characteristics and outcomes in hospitalized COVID-19 patients during the first 28 days of the spring and autumn pandemic waves in Milan: an observational prospective study," Respiratory Medicine, vol. 178, Article ID 106323, 2021. 